\title{
Study on Humidification and Cooling Effect of Garden Plants
}

\author{
Xiaoxia Su ${ }^{1,2}$ \\ ${ }^{1}$ Northeast Forestry University China, 150040, China \\ ${ }^{2}$ Ecological Engineering Vocational College, 150025, China
}

Keywords: humidification, cooling effect, garden plants

\begin{abstract}
In the botanical garden, 14 plant communities with different structural features were selected as research objects. The temperature and humidity were measured by temperature and humidity recorder to study the correlation between canopy density, average crown width, leaf area index and cooling and humidifying effects. Statistical analysis of the experimental data shows that the canopy density is significantly positively correlated with the community cooling effect, and positively correlated with the humidification effect but not significant; the average crown amplitude is significantly positively correlated with the cooling effect of the community, but with the humidification effect. The positive correlation is not significant; the leaf area index plays a certain role in promoting the cooling effect of the community, but has little effect on the humidification effect. In addition, other structural characteristics that affect the cooling and humidifying effects of plant communities need to be further explored.
\end{abstract}

\section{Introduction}

With the rapid development of urbanization, urban land is becoming increasingly tense. How to optimize the spatial layout and structure of urban green space in the limited land resources of the city, give full play to the comprehensive functions of green space, coordinate the relationship between urban development and environmental construction, and realize the city. Sustainable development has become an urgent problem to be solved. Through transpiration, absorption, adsorption, reflection and other functions, garden plants reduce temperature, increase humidity, fix carbon and release oxygen, and resist pollution (absorbing dust, $\mathrm{Cl}_{2}, \mathrm{SO}_{2}, \mathrm{CO}$, etc.), improving and protecting urban environment and ecology. Therefore, the urban green space of the city is an important part of the urban landscape system, and its ecological function is irreplaceable in the urban ecosystem. At present, studying the ecological and environmental benefits of urban green space has become a hot spot in landscape ecology, urban garden ecology and environmental science.

The characteristic factors affecting the ecological benefits of urban green space mainly include green area, green land shape (aspect ratio, height, boundary curvature, perimeter area ratio, etc.), green space landscape structure, green space internal composition, vegetation index, biomass and other landscape ecology. At the same time, the types of garden plants used in urban green space are increasing, and their own environmental and ecological functions are quite different. The evaluation of ecological functions of garden plants and excellent screening should be based on scientific research methods. The research method for the ecological function of humidifying and cooling trees in garden trees is based on the selection of the leaves at the edge of the canopy for measurement. Due to the influence of canopy texture and environmental factors, there are some differences in the benefits of humidification and cooling of leaves in different parts of the canopy. Therefore, this paper attempts to analyze the variation of the humidification and cooling of the leaves in different parts of the canopy by measuring the humidification and cooling of the leaves in different parts of the canopy of 9 garden trees, and to compare the variation of the humidification and cooling of the leaves in different parts of the canopy. The sampling method of ecological function evaluation provides a basis for the selection of excellent tree species for humidification and cooling of garden trees and the evaluation of environmental ecological functions. 


\section{Materials and Methods}

According to the principle of "typicality, representativeness and consistency", 14 representative tree plant communities were selected in Beijing Botanical Garden for temperature and humidity measurement. The selected plant communities are all used in Beijing with high frequency of landscaping, and people can enter freely, and the characteristics of tree species and canopy closure of each community are different.

The most obvious season for plant community cooling and humidification is summer, so this experiment was chosen from July to August. A sample of $10 \mathrm{~m} \times 10 \mathrm{~m}$ is divided into each experimental community selected, and 9 measuring points are set in each sample according to the uniform arrangement, and the surrounding environment is basically the same, and the communities do not affect each other. . In the high temperature weather with similar temperature conditions, the temperature and humidity of the selected community were measured simultaneously using the JL-17 temperature and humidity recorder from 8:00 to 18:00. One community was measured every day, and the instrument recording interval was set to $1 \mathrm{~min}$. The height is $1.5 \mathrm{~m}$ and another control point is set. In this paper, the cooling and humidification effects are defined as the daily average temperature difference, the cooling rate, the daily average humidity difference, and the humidification rate.

Daily average temperature difference $=$ daily average temperature of control points - daily average temperature in the community

Cooling rate $=($ control point temperature - temperature inside the community $) /$ control point temperature $\times 100 \%$

Daily average humidity difference $=$ daily average humidity in the community - daily average humidity of the control point

Humidification rate $=$ (humidity in the community - control point temperature) $/$ control point humidity $\times 100 \%$

The experimental data were correlated with the cooling and humidifying effects of plant communities using SPSS 17.0 software and plotted in EXCEL 2010.

\section{Results and Analysis}

The transpirational heat of plant communities and the occlusion of sunlight by the canopy make the green space a relatively low temperature space. The cold air pressure in the green space is high, the high temperature outside the green space forms a low pressure, and the air flows from the high pressure zone to the low pressure zone, so the cold air is green space flows to the outside. It can be seen from Fig. 1 that each plant community shows a certain cooling effect $(\triangle T>0)$, but the structural parameters such as canopy density, average crown width, leaf area index and heat dissipation of each physiological activity of each community are Different, so there is a certain difference in the cooling effect. The most effective cooling ability was the Peach Peach community, with an average daily temperature drop of $10.88{ }^{\circ} \mathrm{C}$ and a cooling rate of $30.62 \%$, followed by Yangbai Wax, Eucommia ulmoides Oliv., Pinus tabulaeformis, and Hybrid Liriodendron, which were cooled by 27.11\%, 26.53\%, 25.43\%, and 25.31\%, respectively. The plant communities with better cooling effect have greater canopy density and thicker branches and leaves, which is beneficial to the heat dissipation of plant transpiration, so the temperature in the community is significantly lower than the control point. Ginkgo biloba, Tamarix chinensis, Robinia pseudoacacia, eucalyptus, and Paulownia tomentosa communities have poor cooling ability, and the cooling rate is less than $20 \%$. The worst is ginkgo, which is only $4.59{ }^{\circ} \mathrm{C}$, and the cooling rate is only $13.15 \%$.

Plant communities can reduce the temperature of garden green space in summer, thus improving the surrounding small environment, but the internal structure of plant communities is different, and the cooling effect is also different. Correlation analysis was carried out on the effects of canopy density, average crown width, leaf area index and community cooling effect.

There is a significant positive correlation between the cooling effect and the canopy closure of the community. The correlation coefficient between the cooling effect and the canopy closure of the 
community was 0.645 , and the correlation regression results were significant $(\mathrm{P} \leq 0.01)$, indicating that there was a strong correlation between the community cooling rate and the canopy closure. In addition, the community cooling rate and the canopy density showed a positive correlation, which indicates that the increase in canopy density has played a positive role in the cooling rate. That is, the greater the degree of canopy closure, the more solar radiation is blocked, reducing the amount of solar radiant heat entering the community, causing the temperature within the community to decrease. It can also play a role in reducing the internal temperature of the community.

There is a significant positive correlation between the cooling effect and the average crown of the community. Since the correlation coefficient between the average crown width and the community cooling rate is 0.533 , and the F-test is passed at the significant level of $5 \%$, the community cooling rate and the average crown amplitude reach a significant level $(\mathrm{P} \leq 0.05)$. The regression coefficient between the temperature drop rate and the average crown width in the community is 1.952 , so the temperature drop rate in the community is significantly positively correlated with the average crown width. As the average crown width of the community increases, the greater the degree of occlusion of the ground in the community, which can effectively reduce the reflection of direct sunlight and surrounding buildings, greatly reduce the temperature inside the community, and increase the cooling rate of the community.

The cooling effect is positively correlated with the leaf area index of the community. $\mathrm{R}=0.433$, so the leaf area index is positively correlated with the cooling rate, but it is not significant. The reason for this result is that the cooling effect of green space is realized by the function of plant transpiration, reducing radiation balance and reducing the amount of turbulent heat exchange. Therefore, the leaf area index has a certain effect on the cooling effect of the community, but both. The correlation coefficient did not reach a significant level.

Plant communities have obvious moisturizing effects, but the humidification effects of different communities are different. Among the 14 plant communities studied in this paper, the Peach Peach Community had the best moisturizing effect, reaching $84.85 \%$. The other plant communities with humidification rate above $65 \%$ were hybrids Liriodendron, Eucommia ulmoides, Platycladus orientalis, and Pinus tabulaeformis. The humidity increase values were 69.91\%, 69.22\%, 69.11\%, and $67.41 \%$, respectively. In addition, the humidification effect of Tamarix chinensis, Eucalyptus, Paulownia and Ginkgo biloba community was relatively poor, and the lowest moisture-increasing rate was Tamarix chinensis community, which was only $34.59 \%$. According to the comprehensive analysis, the canopy structure of the communities such as Peach, Hexaphylla and Hybrid Liriodendron with good moisturizing effect is more complex than the willow and eucalyptus with poor humidification effect. At the same time, the plant community has better humidification effect. It also shows that their transpiration is more active, and more water is lost through transpiration.

Plant communities can increase air humidity by shading, transpiration, etc., but this humidification will change due to different tree species, canopy structure, and even different time periods. The most active factor is the structure of the plant community itself. To explore the correlation between canopy density, average crown width, leaf area index and community humidification effect, it is of great significance to give full play to the humidification ability of plant communities and improve the living environment.

The humidification effect is positively correlated with the canopy density of the community. The correlation coefficient between community humidification effect and canopy closure is 0.512, which further indicates that the planting community's humidification effect has a positive correlation with its canopy density, which is similar to the results of most scholars in China. As the canopy density of the community increases, the transpiration of the community will increase, transforming the water absorbed by the roots into water vapor, evaporating into the atmosphere through the pores of the leaves, and the moisture emitted by the transpiration will not be due to the large degree of canopy closure. It is quickly lost to the outside environment, thus keeping the humidity in the community at a good level. Therefore, when a large number of plants gather together, it will have an effect on the humidity of the air.

The humidification effect is positively correlated with the average crown width of the 
community. The correlation coefficient and regression coefficient of the average crown width were 0.466 and 5.606, respectively. Therefore, there was a positive correlation between the community humidification rate and the average crown width, but it was not significant. Generally, as the average crown increases, the humidification rate of the community will increase, but the correlation between them is not tight. Therefore, in order to create a good community environment, the proportion of larger tree species in the crown can be appropriately increased, so that the other factors can be combined to make the humidification effect in the community more obvious.

Leaf area index has little effect on the humidification rate in the community. Correlation analysis between leaf area index and its humidification effect is carried out. Because the correlation coefficient is 0.397 and $\mathrm{P}>0.05$, it can be considered that the leaf area index of the community has little effect on the humidification rate, indicating that the leaf area index of the community is humidifying effect. It is still difficult to play a role. Mainly because there is a certain error in the measurement of leaf area index, and the community humidification effect is mainly restricted by plant transpiration. The difference in plant transpiration is not only related to its physiological characteristics, but also affected by environmental temperature and humidity, soil temperature and humidity, Transpiration time and other factors are constrained.

The humidification effect is closely related to the cooling effect. The correlation coefficient between plant community humidification effect and cooling effect was 0 . 920, and the correlation reached a significant level $(\mathrm{P}<0.01$ ), indicating that there is a close relationship between community humidification effect and cooling effect. Due to the transpiration of plant communities, the water vapor content in the air increases, and the relative humidity of the air increases accordingly. The large amount of heat consumed in the vaporization process reduces the air temperature. Therefore, the community cooling effect shows an increasing trend with the increase of the humidification effect.

\section{Conclusion}

Factors such as temperature and humidity, soil moisture, and plant transpiration of the environment affect the temperature and humidity of the microenvironment around the plant to varying degrees. The data obtained in this study are only the relative values of the humidification and cooling effects of monomer plants. Transforming performance to improve the comfort of the human body is still the direction of future research.

\section{References}

[1] Zheng X P. Beijing Urban Green Space System Function Evaluation and Development Strategy. Beijing: Beijing Forestry University, 2011.

[2] Su Y X, Huang G Q, Chen X Z, Chen S S, Li Z S. research progress in the eco-environmental effects of urban green spaces. Acta Ecologica Sinica, 2011, 31(23): 7287-7300.

[3] Chen J, Shi H W, Liao J X, Duan Q M. Studies on the characteristics of photosynthesis and transpiration of 49 garden species in spring in Wuhan city, China. Journal of He' nan University: Natural Science, 2013, 43(1): 62-68.

[4] Chen S P, Zhuang Q Q, Guo T J, Dai X Z, Wang Y. The study of landscape trees' solid carbon and releasing oxygen, humidification cooling effect in Changchun. Hubei Agricultural Sciences, 2012, 51(4): 750-756.

[5] Zheng P, Shi H W, Deng H B, Liao J X. Study on the ecological functions of sixty-five garden species in Wuhan city, China. Plant Science Journal, 2012, 30(5): 468-475. 